\title{
Data reduction in flash method thermography
}

\author{
by P. G. Berardi and G. Cuccurullo \\ Dipartimento di Ingegneria Meccanica, Università di Salerno,, \\ Via Ponte Don Melillo, 84084 Fisciano (SA), E-mail: cuccuru@unisa.it
}

\begin{abstract}
:
An infrared thermography equipment is used to measure the temperature rise at the rear surface of a sample submitted to the pulse irradiation of a flash source, in order to derive simultaneously two thermal properties of the sample. The Levemberg Marquadt technique is used to match the experimental data to the analytical model. Care is taken to tune sample features and the experimental set-up. Since the acquisition time revealed to be a critical parameter, its influence on the final results is investigated too.
\end{abstract}

\section{Introduction}

The flash method is extensively used for measuring the solids thermal diffusivity. The method essentially consists in measuring the surface temperature rise of a thin cylindrical specimen when the other face is radiated by energy pulse. The experimental data compared with the predictions of the theoretical model allow to determine the thermal diffusivity. In origin the method foresaw the use of one or few points of the temperature-time curve, [1]. Actually, the amount of experimental data can be very large, e.g. thermographic systems are available with a scan rate of $50 \mathrm{~Hz}$.

Following the idea of the flash method, different models and data reduction techniques have been proposed, [2-8]. The data reduction method seems to play a significant role in the determination of the thermal diffusivity, in particular the non-linear $\chi^{2}$ fit based on the Levemberg Marquadt technique is shown to lead to the most precise results, [9].

In this work, using the above mentioned fitting technique, the simultaneous identification of the thermophysical properties, i.e. thermal diffusivity and conductivity, by the classical rear-face flash method is attempted. Particular care was taken about the choice of a proper acquisition time by simulating thermograms with Montecarlo tecnique. The influence of sample features on the results is analysed taking into account infrared thermography equipment features. Finally, in order to validate both the experimental procedure and the identification routine, first tests were carried out on certified Teflon samples.

\section{Basic equations and analytical solution}

The identification of the thermophysical properties from the temperature-time profiles needs a model for the heat diffusion: a thin sample subjected to a flash heating and cooled on both faces by radiative-convective heat transfer is considered. The energy balance equation and the related boundary conditions can be written in dimensionless form as:

$$
\begin{aligned}
& \theta^{*} ; \tau=\theta^{*} ; \xi \xi \\
& \theta^{*}\left(0<\xi<\xi_{\text {rif }}, \tau=0\right)=1 / \xi_{\text {rif }} \quad \theta^{*}\left(\xi>\xi_{\text {rif }}, \tau=0\right)=0 \\
& \theta^{*} ;\left.\xi\right|_{\xi=0, \tau}=\left.\operatorname{Bi~} \theta^{*}\right|_{\xi=0, \tau} \\
& \theta^{*} ;\left.\xi\right|_{\xi=1, \tau}=-\left.\operatorname{Bi} \theta^{*}\right|_{\xi=1, \tau}
\end{aligned}
$$

where: $\xi=x / L, x$ being the coordinate through the sample of thickness $L ; \tau=\alpha t / L^{2}, \alpha$ being the thermal diffusivity and the time; $\theta^{*}=\left[\mathrm{T}(\mathrm{x}, \mathrm{t})-\mathrm{T}_{\mathrm{a}}\right] / \Delta \mathrm{T}_{\text {rif }}, \mathrm{T}_{\mathrm{a}}$ being the ambient temperature 
and $\Delta \mathrm{T}_{\text {rif }}=\mathrm{q}_{\mathrm{abs}} /(\rho \mathrm{cL})$ is the equilibrium temperature rise for an adiabatic slab due to the pulse of radiant energy, $\rho c$ being the volumetric heat capacity and $q_{a b s}$ the energy absorbed at wall; $\left(x_{\text {rif }} / L\right) \Delta T_{\text {rif }}$ is the surface temperature rice in a small depth $x_{\text {rif. }} ; \quad B i=h L / k$ is the Biot number, $\mathrm{k}$ being the thermal conductivity and $\mathrm{h}$ the heat transfer coefficient, assumed to be the same on both surfaces.

The unsteady temperature distribution can be obtained in closed form by variation of parameters:

$$
\vartheta^{*}(\xi, \tau, B i)=\frac{T(x, t)-T_{a}}{\Delta T_{\text {rif }}}=\sum_{m=0}^{\infty} C_{m}\left[\lambda_{m} \cos \left(\lambda_{m} \xi\right)+B i \sin \left(\lambda_{m} \xi\right)\right] e^{-\lambda_{m}^{2} \tau}
$$

The characteristic equation and the constants $\mathrm{C}_{\mathrm{m}}$ are:

$$
\frac{\sin \left(\lambda_{m}\right)}{\cos \left(\lambda_{m}\right)}=\frac{2 B i \lambda_{m}}{\lambda_{m}^{2}-B i^{2}} \quad C_{m}=\frac{2 \lambda_{m}}{\lambda_{m}^{2}+2 B i+B i^{2}}
$$

In fig. 1 the temperature evolution of the rear surface, $\theta^{*} ;_{1}(\tau, B i)=\theta^{*}(\xi=1, \tau, B i)$, is shown for different Biot numbers. For the limiting case of an adiabatic slab $(\mathrm{Bi} \rightarrow 0)$ the maximum temperature rise is obtained for $\tau \rightarrow \infty$ and approaches to one.

The asymptotic solution for $\tau>>\tau_{\max }$, i.e. the first term of the summation, is also evidenced with the dash-dot line; in particular, the exponential limiting solution shows that, for $\mathrm{Bi}<<1$, the temperature rise will depend on a single parameter: $\left(\alpha / L^{2}\right) \mathrm{Bi}$ :

$$
\vartheta_{1}^{*}\left(\tau \gg \tau_{\max }, B i<<1\right)=\frac{T_{1}(t)-T_{a}}{\Delta T_{\text {rif }}} \rightarrow\left(1-\frac{13}{288} B i^{2}+\ldots\right) \exp \left(-\frac{(-12+B i+\ldots .)^{2}}{72} B i \frac{\alpha}{L^{2}} t\right)
$$

Having in mind experimental testings based on flash method in order to measure the thermal conductivity and diffusivity by means of temperature-time curves, the analytical solution requires several additional parameters to be known: $T_{a}, q_{a b s}, L$ and $h$. In addition, for performing the data reduction a further parameter is needed: the data reduction time, $t_{\mathrm{RD}}$. The choice of the above parameters will affect the accuracy of the final results in terms of $\alpha$ and $\mathrm{k}$, except the ambient temperature, i.e. the temperature level at which the unknown parameters will be estimated.

The absorbed energy can be controlled but it is difficult to measure, thus it is convenient to work with a new dimensionless temperature normalized with respect to its maximum value:

$$
\vartheta_{1}(\tau, B i)=\frac{\vartheta_{1}^{*}(\tau, B i)}{\vartheta_{1, \max }^{*}(B i)}=\frac{T_{1}(t)-T_{a}}{\Delta T_{1, \max }}
$$

the corresponding plots are shown in fig. 2.

The measure of the heat transfer coefficient is always a critical point due to the radiative and convective contributions; the radiative flux can be controlled by painting the specimens, while the convective one employing a fan. Thus, the overall heat transfer coefficient can be measured by preliminary testing on materials whose properties are certified.

In summary, the parameters that can be handled in experiment testing are: $\Delta \mathrm{T}_{1, \max }, \mathrm{L}$ and $t_{R D}$, the maximum temperature rise, the specimen thickness and the data reduction time. These parameters and the two unknown ones, according to the model, are combined such as: $\Delta T_{1, \max }, t_{R D}, \alpha / L^{2}$ and $L / k$, i.e. the Biot number.

\section{Data reduction}

The accuracy of the results of the data reduction is related to the parameter sensitivity of 
the solution in the time domain. The parameter sensitivity can be described, in a first attempt, by the normalized sensitivity coefficients, defined as: $S_{\beta}=\beta(\partial \mathrm{T} / \partial \beta), \beta$ being a parameter; the parameters will be not correlated if the coefficients are be linearly independent.

Fig. 3 shows the normalized sensitivity coefficients $S_{\alpha / L^{2}}$ and $S_{B i}$, for $\alpha_{\text {ref }} / L^{2}{ }_{\text {ref }}=0.1 s^{-1}, B i_{\text {ref }}$ $=0.1$, which lead to $\tau_{\max }=0.46$. The plots show that the unknown parameters are strongly correlated for $\tau>\tau_{\max }$ and that for $\tau<\tau_{\max }$ the sensitivity to the Biot number is quite small with respect to thermal diffusivity. The behavior for $\tau<\tau_{\max }$ could be expected by looking at the plots of fig. 1 which turn out to be practically independent of the Biot number. On the other hand, for $\tau>\tau_{\max }$, the first term of summation becomes dominant and the solution essentially depends on the product $\alpha \mathrm{Bi}$, as mentioned before.

Due to the non linearity of the unknown parameters provided by the model, the Levenberg-Marquardt (LM) technique has been chosen as data reduction method. this technique is based on minimizing the $\chi^{2}$-merit function:

$$
\chi^{2}(\mathbf{a})=\sum_{i=1}^{N}\left[\frac{y_{i}-y\left(x_{i}, \mathbf{a}\right)}{\sigma_{i}}\right]^{2}
$$

each of the $N$ data points, $\left(y_{i}, x_{i}\right)$, is weighted with its own standard deviations, $\sigma_{i} ; \quad y(x, a)$ is the functional relationship given by the model, where $\mathbf{a}=\left(a_{1}, a_{2 .} . a_{M}\right)$ is the unknown parameters vector. The LM technique encompass both the steepest descent and the inverse-Hessian method, realizing a continuous switching between them, depending on being far or close to the minimum, respectively, [10]. The inverse Hessian method requires :

$$
\mathbf{A} \delta \mathbf{a}=\mathbf{b}
$$

where: $\delta \mathbf{a}=\mathbf{a}_{\text {nex }}-\mathbf{a}_{\text {curr }} ; \mathbf{b}=-\nabla \chi^{2} \mid \mathbf{a}_{\text {curr }}$ is the gradient of $\chi^{2} ; \mathbf{A}$ is the Hessian square $M \times M$ matrix given by:

$$
\frac{\partial^{2} \chi^{2}}{\partial a_{k} \partial a_{j}}=2 \sum_{i=1}^{N} \frac{1}{\sigma_{i}^{2}} \frac{\partial y\left(x_{i}, \mathbf{a}\right)}{\partial a_{k}} \frac{\partial y\left(x_{i}, \mathbf{a}\right)}{\partial a_{k}}-2 \sum_{i=1}^{N} \frac{y_{i}-y\left(x_{i}, \mathbf{a}\right)}{\sigma_{i}^{2}} \frac{\partial^{2} y\left(x_{i}, \mathbf{a}\right)}{\partial a_{k} \partial a_{j}}
$$

Note that the term containing the second derivative can be cancelled, since the measurement error at each point is random.

The steepest descent method can be put in the same fashion of the above equation if $\mathbf{A}$ is a constant, i.e. $\mathbf{A}$ is a diagonal matrix.

A dimensionless parameter $\gamma$ is used to weight the diagonal term of the Hessian such as the steepest descent and the inverse-Hessian method are recovered for $\gamma->\infty$ and $\gamma->0$, respectively. During the iterative process toward the minimum, proper values of the dimensionless parameter $\gamma$ allow to switch between the two minimization methods.

To control the accuracy of the LM technique in estimating the two unknown thermal parameters, preliminary tests were performed on Montecarlo simulated thermograms, obtained by perturbing the analytical temperature profiles. In fig. 4 , a basic temperature profile and the corresponding perturbed one are shown up to a dimensionless observation time $\tau_{\text {obs }}=6 \tau_{\text {max }}$, for $\alpha_{\text {ref }} / L^{2}$ ref $=0.1 \mathrm{~s}^{-1}, B i_{\text {ref }}=0.1$ and $\tau_{\max }=0.46$ and assuming an uncertainty equal to $+/-0.05$.

To process the simulated data, increasing data reduction times are chosen starting from the beginning of the phenomenon; in particular $\tau_{R D, n}=(n / 12) \tau_{\text {obs }}$, with $n=1,2, . .12$, see fig. 4 . One hundred simulated temperature profiles were performed and, for each data reduction time, the values of $\alpha / L^{2}$ and $\mathrm{Bi}$ are obtained, searching for the minimum of the $\chi^{2}$-merit function. The average values and the related standard deviations are plotted in fig. 5 and 6 , 
in terms of $\alpha / \alpha_{\text {ref }}$ and $\mathrm{k} / \mathrm{k}_{\text {ref, }}$, the slab thickness and the heat transfer coefficient being assumed known. The plots show that for $\tau_{\mathrm{RD}}<\tau_{\max }$, i.e. $\mathrm{n}<2$, the diffusivity can be derived but the Biot number exhibits a great uncertainty, such as evidenced before by the sensitivity coefficients. On the other hand for $\tau_{\mathrm{RD}}>\tau_{\max }$, both thermal diffusivity and Biot number can be evaluated; in particular it seems evident that the data reduction time can be chosen as $\tau_{\mathrm{RD}}=3 \tau_{\max }$, since no meaningful accuracy variation in the target values is attained beyond this limit and unuseful data to process can be avoided. It has to be noted that, in agreement with the sensitivity analysis, the uncertainty on the thermal conductivity is always greater than the one related to the diffusivity.

\section{4. experimental tuning}

The experimental temperature-time curves depend on: two unknown thermal parameters, $\alpha$ and $k$, and three experimental set-up ones, $\Delta T_{1, \max }, L$ and $t_{R D}=3 t_{\max }$, this latter being chosen according to discussion of the previous section.

In order to achieve a preliminar tuning of the experimental set-up, the features of the material to be tested and the main characteristics of the thermographic system are to be taken into account. The choice of the specimen thickness is expected to be a critical step: in fact it influences both the maximum temperature rise and the related time at which it is attained. On the other hand, these latter parameters are also related to the specimen and experimental setup properties. With reference to the thermal properties, these can often be ghly estimated. Also the flash absorbed energy can be estimated by knowing the flash features and by painting the slab surface. The heat tranfer coefficient can be sought to be known, as before mentioned. Finally, $\Delta \mathrm{T}_{1, \max }$, and $\mathrm{t}_{\max }$ depend essentially on the slab thickness and they can be easily calculated according to the model.

In the following, for instance, assuming $\alpha_{\text {ref }}=0.110^{-6} \mathrm{~m}^{2} / \mathrm{s}, \mathrm{k}_{\text {ref }}=10^{-1} \mathrm{~W} /(\mathrm{mK}), q_{\mathrm{abs}}=10^{4} \mathrm{~J} / \mathrm{m}^{2}$ and $\mathrm{h}=10 \mathrm{~W} /\left(\mathrm{m}^{2} \mathrm{~K}\right)$, the variation of $\Delta \mathrm{T}_{1, \max }$, and $\mathrm{t}_{\max }$ with the slab thickness is drawn, see fig. 7. As expected, the plots show that with increasing thickness the maximum temperature rise decreases, while the corresponding time increases.

The choice of the sample thickness must be accomplished so that $\Delta T_{\max }$ and $t_{\max }$ turn out to be not small when compared with temperature and time resolution of the temperature detector; these features will lead to a working range for the slab thickness.

Consider experiments with thermographic systems, which exhibit temperature resolution of $0.05^{\circ} \mathrm{C}$ at ambient temperature and scan rate of $50 \mathrm{~Hz}$. For the above test case, admitting a relative error on temperature contained within $2 \%$, i.e. $\Delta \mathrm{T}_{1, \max }=5^{\circ} \mathrm{C}$, one has to choose thickness at least $1.7 \mathrm{~mm}$. Wishing 50 measures in one second, one has that the thicknes must be greater than $0.4 \mathrm{~mm}$. On the other hand, for $0.4<\mathrm{L}<1.7 \mathrm{~mm}$, no problems exists about the time rate, while the temperature rise is greater than the previous limit and can affect the level at which the thermal properties will be measured; in addition, the overall heat transfer coefficient changes, thus a new calibration is required.

\section{Application}

A thin certified Teflon sample, $\mathrm{L}=2 \mathrm{~mm}$, is submitted to pulse irradiation of a flash. Both base surfaces are black painted, in order to increase infrared emissivity, while the remaining surface is made adiabatic. An air fan stabilizes the heat transfer coefficient on both surfaces to a measured value of $\mathrm{h}=10.7 \mathrm{~W} /\left(\mathrm{m}^{2} \mathrm{~K}\right)$. An INFRAMETRICS SC 9000 infrared thermography equipment is used to measure the temperature rise at the rear surface of the test specimen. The experiments were performed at room temperature and the start time of the transient temperature is directly recorded on thermograms.

First tests were carried out on certified Teflon samples $2 / 3 / 4 \mathrm{~mm}$ thick, obtaining a diffusivity value of $\alpha=(9.4 \pm 0.1) \mathrm{mm}^{2} \mathrm{~s}^{-1}$ and conductivity of $\mathrm{k}=(0.23 \pm 0.02) \mathrm{W} /(\mathrm{mK})$; these 
values agree quite fairly with the declared values.

To restate assessments of the previous sections about the influence of the data reduction time, as an example, the thermogram in fig. 8 is analysed and results are summarized in the following table.

\begin{tabular}{|c|c|c|c|c|c|}
\hline $\mathrm{n}$ & $\mathrm{t}_{\mathrm{RD}} / \mathrm{t}_{\max }$ & $\begin{array}{c}\mathrm{k} \\
\mathrm{W} /(\mathrm{mK})\end{array}$ & $\mathrm{k} / \mathrm{k}_{6}$ & $\begin{array}{c}\alpha 10^{8} \\
\mathrm{~m}^{2} / \mathrm{s}\end{array}$ & $\alpha / \alpha_{6}$ \\
\hline 1 & $1 / 2$ & 0,24 & 1,07 & 9,42 & 1,005 \\
\hline 2 & $2 / 2$ & 0,22 & 0,95 & 9,37 & 0,999 \\
\hline 3 & $3 / 2$ & 0,32 & 1,40 & 9,59 & 1,022 \\
\hline 4 & $4 / 2$ & 0,24 & 1,05 & 9,43 & 1,004 \\
\hline 5 & $5 / 2$ & 0,24 & 1,02 & 9,40 & 1,003 \\
\hline 6 & $6 / 2$ & 0,23 & 1 & 9,38 & 1 \\
\hline
\end{tabular}

The data clearly evidence the behavior discussed in the data reduction section and presented in the figs. 5 and 6; in particular, reduction applied to variable times allows to find practically the final results after a data reduction time of $t_{R D}=2 t_{\max }$; once again, it is evident that the error on the thermal diffusivity is more contained than the one on the thermal conductivity.

\section{References}

[1] PARKER, W. J., JENKINS, R. J., BUTIER, C. P. and ABBOT, G. L., "Flash method for determining thermal diffusivity, heat capacity, and thermal conductivity", J. Appi. Phys., 32, 1961, pp. 1679-1684

[2] GEMBAROVIC, J., VOZÀR, L. and MAJERNIK, V., "Using the least square method for data reduction in the flash method", Int. J. Heat Mass Transfer 33, 1990, pp. 15631565

[3] PAWLOWSKI, L. and FOUCHAIS, P., "The least square method in determination of thermal diffusivity using flash method", Rev. Phys. Appl. 21, 1986, pp. 83-86

[4] TAKAHASHI, Y., YAMAMOTO, K., OHSATO, I. and TERAI, T., "Usefulness of Logarithmic method in Laser-flash technique for thermal diffusivity measurement, Proceedings of the 9th Japanese Symposium on Thermophysical Properties", 1988, pp. 175-178

[5] CLARK, L. M. and TAYLOR, R. E., "Radiation loss in the flash method for thermal diffusivity", J. Appl. Phys. 46, 1975, pp. 714-719

[6] HECKMAN, R. C., "Finite-pulse time and heat loss effects in pulse thermal diffusivity measurements", J. Appl. Phys., 44, pp. 1455-1460

[7] VOZÀR, L., GEMBAROVIC, J. and MAJERNIK, V., "New method for data reduction in flash method", Int. J. Heat Mass Transfer 34, 1991, pp. 1316-1318

[8] SRAMKOVA, T. and TORGRIM, L., "Using non linear $\chi 2$ fit in flash method", Int. J. Heat Mass Transfer 38, 1995, pp. 2885-2891

[9] VOZÀR, L. and SRAMKOVA, T., Two data reduction methods for evaluation of thermal diffusivity from step heating measurements, Int. J. Heat Mass Transfer 40, 1997, pp. 1647-1655

[10] PRESS, W. H., FLANNERY, B. P., TEUKOLSKY, S A. and VETTERLING, W. T., Numerical Recipes in C.", Cambridge 1990, Cambridge University Press, 


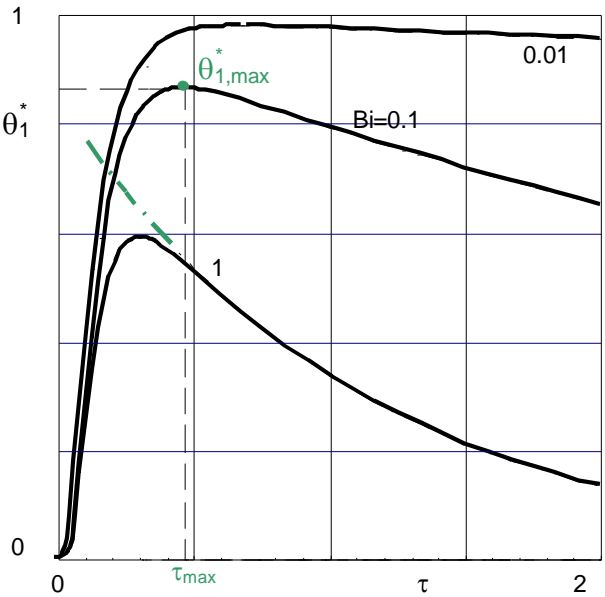

fig. 1 - dimensionless temperature profiles

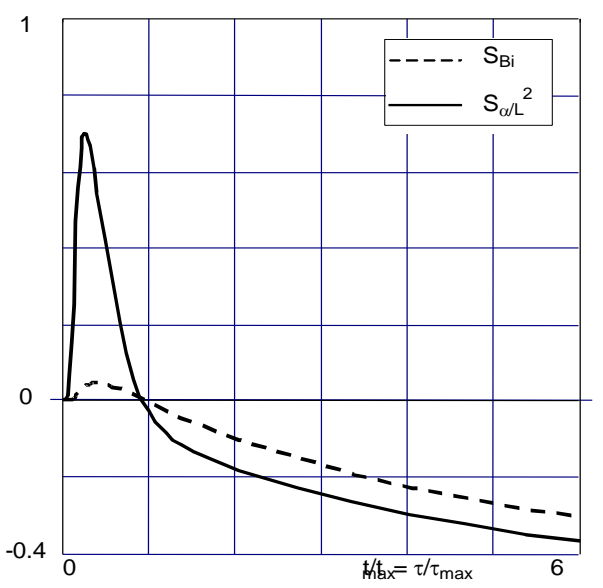

fig. 3 - the sensitivity coefficients

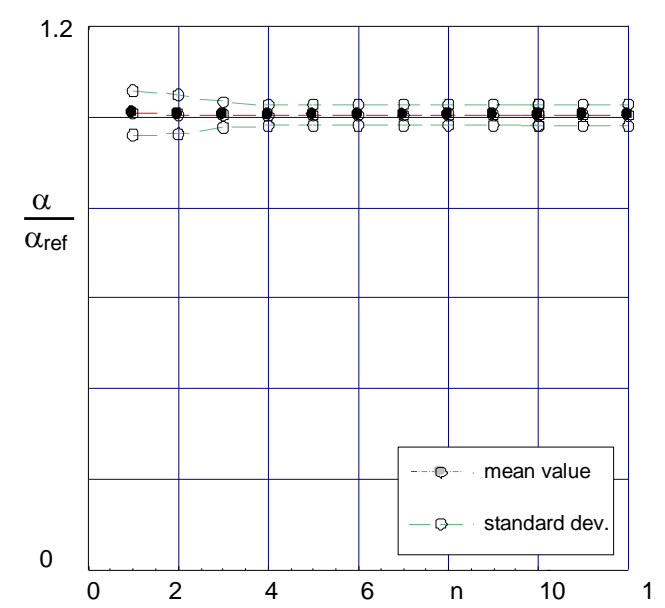

fig. 5 -thermal diffusivity by simulation

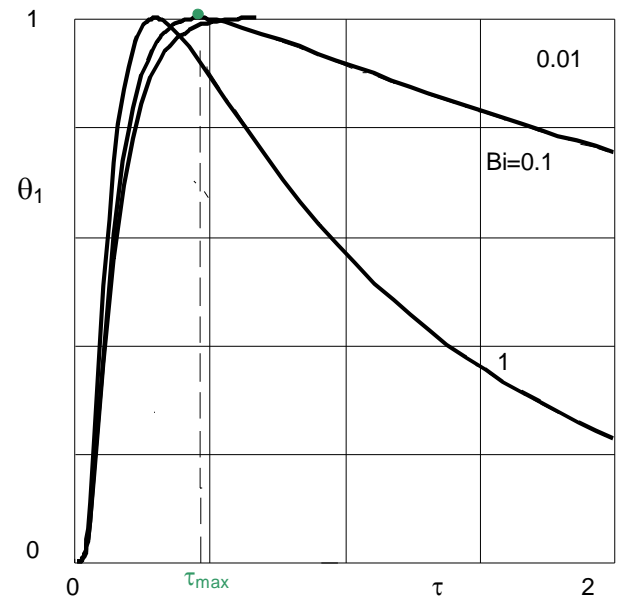

fig. 2 - normalized temperature profiles

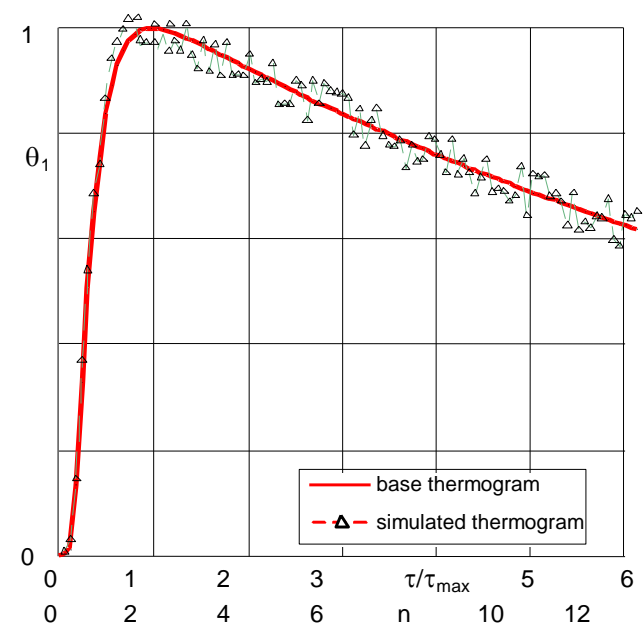

fig. 4 - base and simulated thermograms

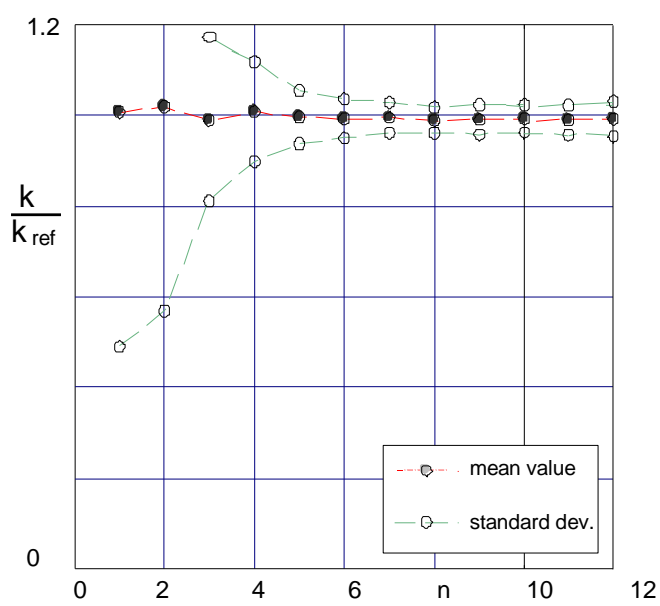

fig.6- thermal conductivity by simulation 
http://dx.doi.org/10.21611/qirt.2000.040

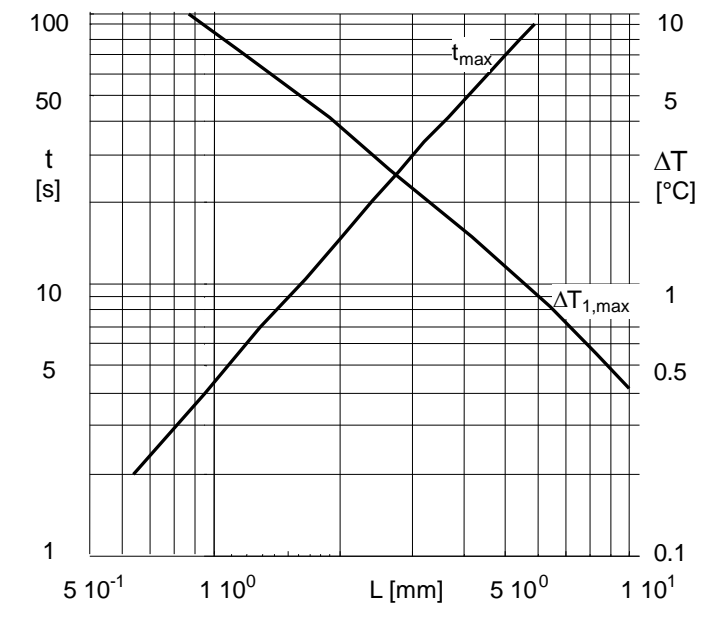

fig. 7- thickness tuning

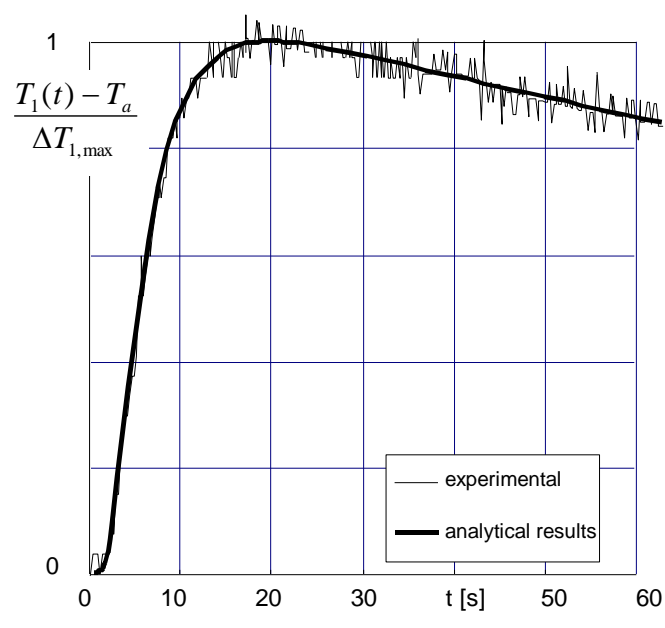

fig. 8- $2 \mathrm{~mm}$ Teflon sample 